RAD Conference Proceedings, vol. 2, pp. 173-177, 2017

www.rad-proceedings.org

salororitt

\title{
TOMOTHERAPY IN UKRAINE - GENERAL ANALYSIS OF FIRST TREATMENT RESULTS
}

\section{S.P. Odarchenko, K.V. Gumeniuk*, M.B. Gumeniuk, O.V. Zinvaliuk, D.S. Synchuk}

\author{
LLC “Ukrainian center of Tomotherapy”, Kropyvnytskyy, Ukraine
}

\begin{abstract}
The work presents first results of radiation therapy with using Tomotherapy TomoHD. For the high quality of patients' treatment, modern methods of radiotherapy, such as IGRT, IMRT and adaptive radiotherapy are used. Regular performing of machine quality assurance procedures are done according to the local program of $Q A$. A good stability of different machine parameters provides a high quality of patients' treatment. After the end of the treatment, the partial resorption of targeted lesions and the absence or minimization of early reactions are observed. Tomotherapy provides a fundamentally new and effective treatment for all cancer locations.
\end{abstract}

Key words: IGRT, IMRT, quality assurance, TomoTherapy Tomo HD, treatment planning, tumor response

DOI: $10.21175 /$ RadProc.2017.35

\section{INTRODUCTION}

In April 2015, the first and only radiotherapy system TomoTherapy TomoHD in Ukraine was installed in Ukrainian center of TomoTherapy (UCT).

TomoTherapy System was developed for the implementation of IMRT and IGRT techniques.

A wide variety of clinical uses of TomoHD machine directly follow from the method of dose delivery, which is carried out by two modes (Helical and Direct).

The TomoHelical delivery mode provides IMRT and ${ }_{3} \mathrm{D}$ CRT treatment delivery in a continuous $\left(360^{\circ}\right)$ helical mode. The TomoHelical mode maximizes conformality and uniformity of tumor dose coverage while minimizing exposure of healthy tissue. During treatment delivery, the linear accelerator makes multiple $360^{\circ}$ rotations around the patient while the couch passes through the bore of the gantry.

The TomoDirect delivery mode is non-rotational delivery mode with a discrete angle static beams ( 2 or more). TomoDirect allows to create treatment plans that include between 2 and 12 target-specific gantry angles. Also it allows the user to define the level of modulation for the plan, including a $3 \mathrm{D}$ delivery mode. During treatment delivery, all beams for each target are delivered sequentially with the couch passing through the bore of the gantry.

This system allows the treatment for a wide range of clinical cases. That could be very small tumor lesions, located in the brain and throughout the body. The system can be used for hypofractionated treatments such as SRS and SBRT. In addition, superficial treatment, for example skull treatment can be performed because of the rotational tangential beam. Also total body irradiation (TBI) or general bone marrow irradiation (TMI) could be realized [1].

In these cases, the use of a conventional linear accelerator requires special therapeutic procedures, often involving the modification of radiation geometry (using non standard distances, specifically custom designed couches for the treatment, etc.).

This article includes methods of dose delivery, treatment plans requirements, quality control of therapeutic plans, evaluation of tumor responses to the treatment, analysis of radiotherapy systems work and quality control of machine characteristics.

\section{MATERIALS AND METHODS}

In the newest external beam radiotherapy system TomoTherapy TomoHD the linear accelerator (linac) is mounted on a CT scanner-like ring gantry, which means TomoTherapy treatments can be delivered continuously, from all angles around the patient [2].

The dose delivery to the target is carried out in a spiral mode or by using directional beams.

\subsection{Tomotherapy machine - technical parameters}

The TomoTherapy treatment system uses a patented multi-leaf collimator MLC with 64 pneumatically driven leafs, each leaf creates $6.25 \mathrm{~mm}$ wide "beamlet" and is either opened or fully closed. So, MLC divides the radiation beam into beamlets, all aimed at the tumor. Typically, tens of thousands of beamlets are used in a single TomoTherapy treatment session. The powerful software optimizes the contribution of each one to the total tumor dose, minimizing the exposure of the healthy tissue.

\footnotetext{
*ekaterina_goptsy@mail.ru
} 
Tomo HD has two energies - $6 \mathrm{MV}$ for the treatment and 3.5 MV for CT (MVCT). For a different plan the field width can be $1 ; 2.5$ or $5 \mathrm{~cm}$ (3 clinical beams are available: $1 \times 40,2.5 \times 40$ and $5 \times 40 \mathrm{~cm}$ ). Long therapeutic field $40 \mathrm{~cm}$ (transverse diameter) x $135 \mathrm{~cm}$ (longitudinal) may be calculated for a typical patient.

The SAD is shorter $(85 \mathrm{~cm})$ then in conventional linacs. A very important aspect of Tomotherapy design is the absence of a flattening filter. The lack of the flattening filter results in a higher dose rate (nominally $850 \mathrm{cGy} / \mathrm{min}$ for field $5 \times 40 \mathrm{~cm}$ at $\mathrm{D}_{\max }$ ). The system comes pre-commissioned from the factory with a model 6 MV X-ray beam. The user performs the acceptance tests and beam data measurements to verify the model beam parameters. The phantom IMRT plans are measured for on-axis and off-axis targets for validation of the model and the treatment delivery (phantom based test).

The TomoHD System includes stationary green lasers for virtual isocenter (70 $\mathrm{cm}$ from real isocenter) and moveable red lasers for patient positioning and registration. Green laser is typically used by physicists for quality assurance and research procedures.

The position of moveable red lasers is determined by the physicist during planning process. Red lasers can be programmed to go to a patient-specific position. Initial patient setup typically involves aligning patient fiducial marks to red lasers. After scanning, registering, accepting registration results, and applying registration results, red lasers move to account for the registration results.

The inbuilt CTrue ${ }^{\mathrm{TM}}$ (MVCT) visualization system allows to check the position of the patient on a treatment couch on a daily basis by comparing MVCT with CT images, which were used to plan optimization. MVCT doses range from 0.8 cGy to $2.8 \mathrm{cGy}$, depending on the selected scanning mode (image quality is obtained). Tomotherapy imaging detector is a standard CT imaging system. An arc-shaped xenon detector is mounted on the gantry opposite to the linac. The detecting volume is composed of 640 millimetre chambers separated by tungsten plates. Only 520 chambers are used for imaging, the standard image size is $512 \times 512$ pixels and the field of view is $40 \mathrm{~cm}$ wide [3].

\subsection{Tomotherapy treatment planning}

Before the beginning of optimization, the field width $(1,2.5$ or $5 \mathrm{~cm})$, pitch $(0.215,0.287$ or 0.430$)$, delivery mode (Helical or Direct), prescribed dose to the target, calculation grid (Fine: $256 \times$ 256, Normal:128 x 128 or Coarse: 64 × 64) and modulation factor (3.4 at the begining) must be selected. TomoTherapy treatment planning system uses the idea of reverse planning. It means, that till the end of plan optimization the number of beams and their direction are unknown. Three dose calculation methods are available for optimization calculations - terma, scatter and -beamlet mode, which is the most commonly used (good compromise to speed up optimization process). Generation of final dose distribution (patient delivery sinogram) is the result of full superposition of dose calculations including corrections for MLC hardware. For that reason, the next stage of planning, typical for tomotherapy nonVoLO systems, beamlet calculations are made in background mode. It takes different time (30 min - 7 hours) and depends on the calculation accuracy that was selected. A beamlet is an element of a beam emitted through a single open leaf during one projection. Each beamlet is divided into multiple rays to ensure at least one ray per voxel. All possible projections are calculated by the Tomo cluster.

After that, the plan optimization is started and dose distribution, maximums and minimums are controlled by using the dose volume histogram curve (DVH). During the optimization, it is possible to reduce the modulation factor (1.8 to $2.7,2.3$ is the most common MF) thereby reducing the fraction time, but this also may reduce the plan quality.

The patient is irradiated from different directions that provides a homogeneous dose distribution in the tumor with a large gradient at the edges, and also allows minimization the absorbed dose in surrounding healthy tissues.

Taking into account possible changes of healthy organs, tissues and tumor size, treatment plans may be adapted if needed. Adaptive radiation therapy is a process where the treatment plan can be modified and treatment dose can be routinely customized to each individual patient to achieve a safe escalation dose.

\section{RESULTS}

197 patients, who were treated with TomoHD during 2015-2016, were selected for the analysis. The most common treatment sites were lymph nodes - $27 \%$, brain $-20 \%$, prostate $-9 \%$, bones $-9 \%$, breast - $8 \%$ and other localizations - 27\% (Fig.1).

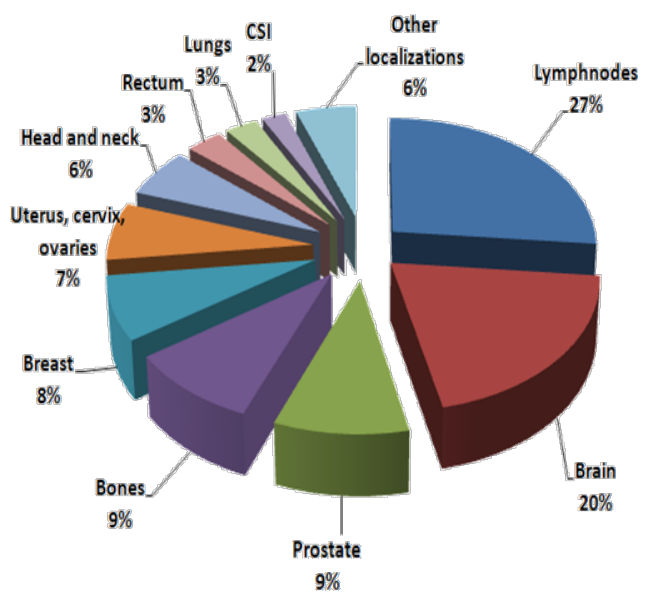

Figure 1. Treatment sites, which were treated with TomoHD during 2015-2016

The dosimetric analysis of plans showed uniform and conformal target coverage in majority of cases.

Analyzing the dosimetry plan, it should be noted that the value of the mean dose Vptv covered 100\% and met the prescribed dose, dose value (Dptv) that 
covered Vptv 99\% (95\% in some complicated cases) was more than 95\%, the maximum dose Dmax $\leq 107 \%$.

Organs at risk received a dose, which did not exceed the tolerance doses according to the recommendations of the QUANTEC protocol [4], except those, that had common points with the target. An average irradiation time was about $420 \mathrm{~s}$.

1. An example of brain tumor irradiation is shown in Figure 2. Prescribed dose was $30 \mathrm{~Gy}$ for whole brain $\mathrm{PTV}_{\text {brain }}\left(\mathrm{PTV}_{1}\right)$ and $40 \mathrm{~Gy}$ for Mts PTV mts $\left(\mathrm{PTV}_{2 \text { plan }}\right)$ per 10 fractions.

The field width for this plan was $2.5 \mathrm{~cm}$, the modulation factor was 2.131 and the pitch was 0.287 . The beam-on time was $378 \mathrm{sec}$. Plan results are shown in tables 1 and 2.
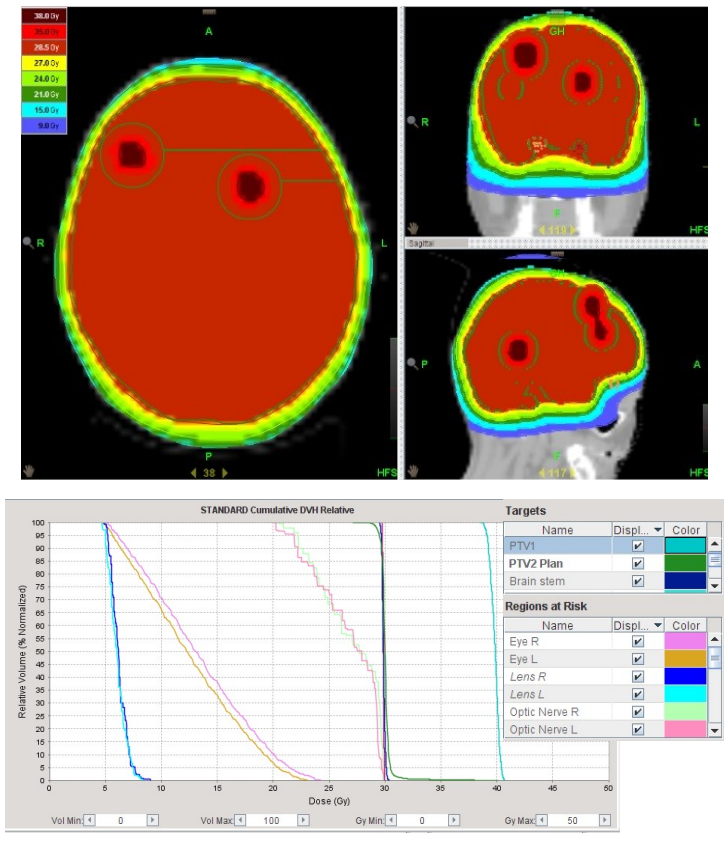

Figure 2. Simultaneous integrated boost case for brain tumors. Dose distribution and OARs doses.

Table 1. PTV DVH characteristics

\begin{tabular}{|c|c|c|c|}
\hline & $\begin{array}{c}\mathrm{D}_{\text {presc }} \\
\text { per 10fr }\end{array}$ & $\begin{array}{c}\mathrm{D}_{\min } / \mathrm{D}_{99 \%}, \\
\mathrm{~Gy}\end{array}$ & $\mathrm{D}_{\max } / \mathrm{D}_{1 \%}, \mathrm{~Gy}$ \\
\hline $\mathrm{PTV}_{\text {brain }}-\mathrm{PTV}_{\mathrm{mts}}$ & $30 \mathrm{~Gy}$ & $27.1 / 29.1$ & $40.1 / 31.4$ \\
\hline $\mathrm{PTV}_{\mathrm{mts}}$ & $40 \mathrm{~Gy}$ & $38.5 / 38.8$ & $40.7 / 40.5$ \\
\hline
\end{tabular}

Table 2. OARs dose characteristics

\begin{tabular}{|c|c|}
\hline Lens R & $\mathrm{D}_{\text {max }}=9 \mathrm{~Gy}$ \\
\hline Lens L & $\mathrm{D}_{\text {max }}=8 \mathrm{~Gy}$ \\
\hline Eye R & $\mathrm{D}_{\text {mean }}=13.1 \mathrm{~Gy}$ \\
\hline Eye L & $\mathrm{D}_{\text {mean }}=12.3 \mathrm{~Gy}$ \\
\hline Optic Nerve L & $\mathrm{D}_{\text {max }}=29.9 \mathrm{~Gy}$ \\
\hline Optic Nerve R & $\mathrm{D}_{\text {max }}=29.8 \mathrm{~Gy}$ \\
\hline Chiasm & $\mathrm{D}_{\text {max }}=29.9 \mathrm{~Gy}$ \\
\hline Brainstem & $\mathrm{D}_{\text {max }}=30 \mathrm{~Gy}$ \\
\hline
\end{tabular}

2. An example of prostate case with Mts irradiation is shown in Figure 3. Prescribed dose was lymph nodes (PTV ${ }_{\mathrm{LN}}$ ) - 54 Gy, lymph nodes Mts (PTV mtsLN) - 64 Gy, prostate $\left(\mathrm{PTV}_{\mathrm{pr}}\right)-73.6$ Gy per 32 fractions.

The field width for this plan was $2.5 \mathrm{~cm}$, the modulation factor was 2.141 and the pitch was 0.287 . The beam-on time was $422 \mathrm{sec}$. Plan results are shown in tables 3 and 4.
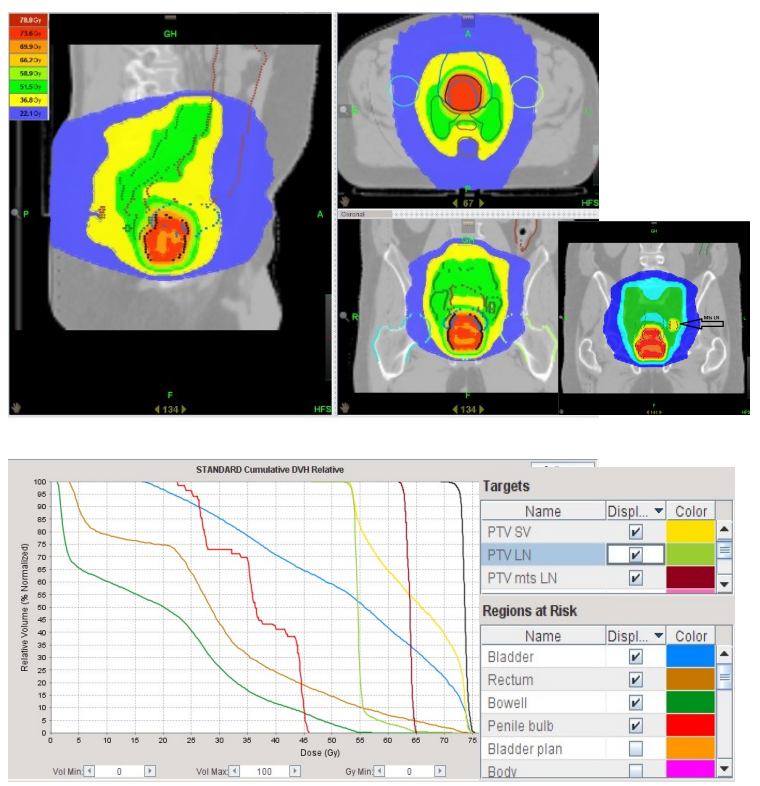

Figure 3. Simultaneous integrated boost case for prostate, lymph nodes, lymph nodes Mts, seminal vesicles. Dose distribution and OARs doses.

Table 3. PTV DVH characteristics

\begin{tabular}{|c|c|c|c|}
\hline & $\mathrm{D}_{\text {presc }}$ per $32 \mathrm{fr}$ & $\mathrm{D}_{\min } / \mathrm{D}_{99 \%}, \mathrm{~Gy}$ & $\begin{array}{c}\mathrm{D}_{\max } / \mathrm{D}_{1 \%}, \\
\mathrm{~Gy}\end{array}$ \\
\hline $\mathrm{PTV}_{\mathrm{LN}}$ & $54 \mathrm{~Gy}$ & $51.2 / 53.3$ & $73.9 / 58.1$ \\
\hline $\mathrm{PTV}_{\mathrm{mtsLN}}$ & $64 \mathrm{~Gy}$ & $61.9 / 62.0$ & $65.1 / 64.8$ \\
\hline $\mathrm{PTV}_{\mathrm{Pr}}$ & $73.6 \mathrm{~Gy}$ & $68.4 / 71.0$ & $75.8 / 75.0$ \\
\hline
\end{tabular}

Table 4. OARs dose characteristics

\begin{tabular}{|c|c|c|c|c|}
\hline OARs & $\mathrm{D}_{\mathrm{V}_{15} \%}$ & $\mathrm{D}_{\mathrm{V}_{25 \%}}$ & $\mathrm{D}_{\mathrm{V}_{35 \%}}$ & $\mathrm{D}_{\mathrm{V}_{50 \%}}$ \\
\hline Rectum & $50 \mathrm{~Gy}$ & $40 \mathrm{~Gy}$ & $33 \mathrm{~Gy}$ & $29 \mathrm{~Gy}$ \\
\hline Bladder & $72 \mathrm{~Gy}$ & $68 \mathrm{~Gy}$ & $64 \mathrm{~Gy}$ & $56 \mathrm{~Gy}$ \\
\hline Femoral head L & - & $22.6 \mathrm{~Gy}$ & - & - \\
\hline Femoral head R & - & $22.0 \mathrm{~Gy}$ & - & - \\
\hline
\end{tabular}

3. An example of CNS case irradiation is shown in Figure 4. Prescribed dose for target was 36 Gy (PTV $\left.{ }_{\text {general }}\right)$ per 20 fractions.

The field width for this plan was $5 \mathrm{~cm}$, the modulation factor was 2.321 and the pitch was 0.303 . The beam-on time was $728 \mathrm{sec}$. Plan results are shown in tables 5 and 6. 

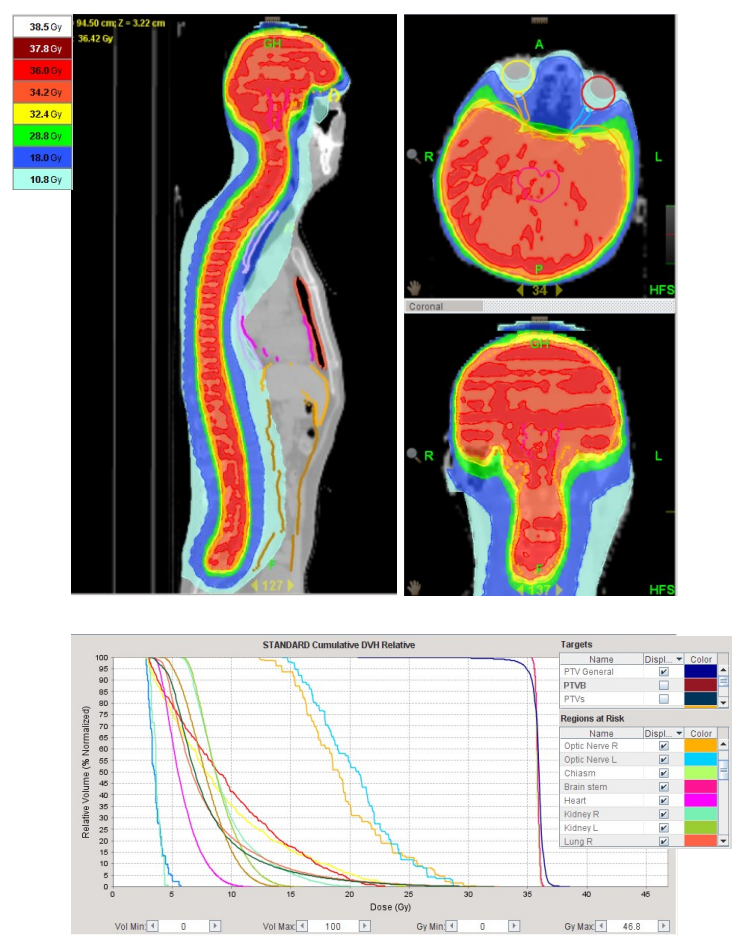

Figure 4. Craniospinal irradiation. Dose distribution and OARs doses.

Table 5. PTV DVH characteristics

\begin{tabular}{|c|c|}
\hline PTV D $_{\min } / \mathrm{D}_{\mathrm{V}_{99} \%}, \mathrm{~Gy}$ & PTV D $_{\max } / \mathrm{D}_{\mathrm{V}_{1} \%}, \mathrm{~Gy}$ \\
\hline $20.9 / 33.5$ & $38.7 / 36.9$ \\
\hline
\end{tabular}

Table 6. OARs dose characteristics

\begin{tabular}{|c|c|}
\hline Lens R & $\mathrm{D}_{\text {max }}=5.9 \mathrm{~Gy}$ \\
\hline Lens L & $\mathrm{D}_{\text {max }}=4.9 \mathrm{~Gy}$ \\
\hline Optic Nerve L & $\mathrm{D}_{\text {max }}=31.1 \mathrm{~Gy}$ \\
\hline Optic Nerve R & $\mathrm{D}_{\text {max }}=29.0 \mathrm{~Gy}$ \\
\hline Chiasm & $\mathrm{D}_{\text {max }}=36.4 \mathrm{~Gy}$ \\
\hline Brainstem & $\mathrm{D}_{\text {max }}=36.5 \mathrm{~Gy}$ \\
\hline Lung R & $\mathrm{D}_{\mathrm{V}_{20} \%}=9.9 \mathrm{~Gy}$ \\
\hline Lung L & $\mathrm{D}_{\mathrm{V}_{20} \%}=10.5 \mathrm{~Gy}$ \\
\hline Kidney R & $\mathrm{D}_{\text {mean }}=9.0 \mathrm{~Gy}$ \\
\hline Kidney L & $\mathrm{D}_{\text {mean }}=9.0 \mathrm{~Gy}$ \\
\hline
\end{tabular}

The risk of inadequate radiation treatment is minimized through the delivery quality assurance (DQA) procedures performing and systematic execution of a comprehensive Quality Assurance (QA) program. For DQA PTW Octavius II with Octavius detector 729 and PTW VeriSoft were used. The regular pre-treatment dose verification shows results within 95\% -100\% (analysis with the following parameters: Gamma 3D, $2 \mathrm{~mm}$ distance to agreement / 2 \% dose difference with reference to the local dose. Criteria $3 \mathrm{~mm} / 3 \%$ were used for complicated cases).

Quality Assurance (QA) program involves programs for quality management and includes periodic quality control of equipment. An automated hardware control is provided by the inbuilt TomoTherapy Quality 176
Assurance (TQA) software. Also independent quality control of TomoHD characteristics is performed with the set of special dosimetric equipment and phantoms according to local protocols and Report of the AAPM Task Group 148 recommendations [5]. The analysis of periodic quality assurance results shows that the output, energy and other parameters of TomoTherapy unit are quite stable. The results are consistent between the different measurement tools and techniques, proving not only the dosimetric, but also mechanical stability. That stability of different machine parameters provides a high quality of patient's treatment during the course.

The evaluation of the tumor response was carried out according to RECIST 1.1. Complications were assessed with CTCA 4.03 side effects staging [6]. Treatments were performed according to local protocols.

The analysis of early treatment's results shown that there were $15 \%$ patients with prolongation of the disease; the complete resorption was observed in $10 \%$; stabilization in $19 \%$; partial regression in $56 \%$ of cases (Fig 5).

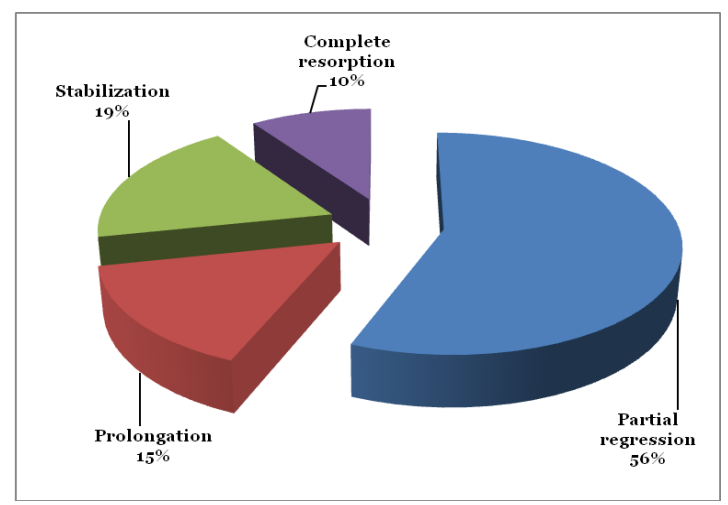

Figure 5. Early treatment's results diagram

There were only few acute radiation reactions which did not exceed G1, G2. The most frequent reactions were dermatitis - 32\%, epithelitis - $14 \%$, leukopenia $-14 \%$, cystitis $-14 \%$, anemia $-8 \%$, rectitis $-6 \%$, esophagitis $-5 \%$, stomatitis $-2 \%$ (Fig. 6 ). There were no interruptions of the treatment course caused by acute reactions.

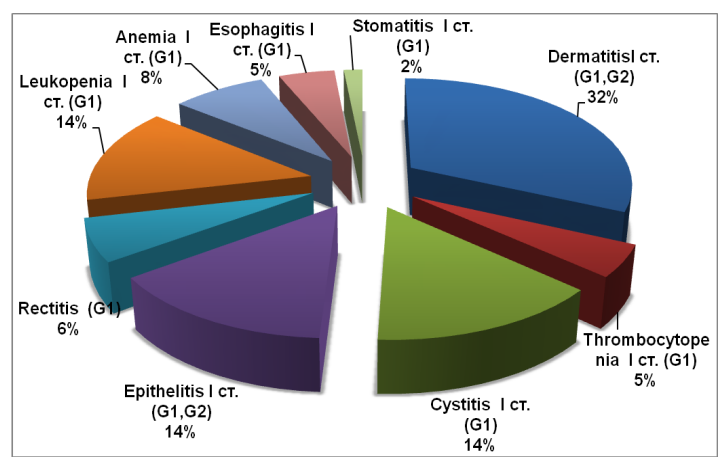

Figure 6. Early radiation reactions results 


\section{CONCLUSIONS}

Helical tomotherapy is one of the radiotherapy treatment methods. This method enables the treatment implementation for a wide spectrum of clinical cases, beginning with very small neoplastic lesions located in the brain or in the whole body, treated with conventional stereotactic methods or radiation surgery, and ending with vast target volumes such as anaplastic medulloblastoma, total body irradiation (TBI) or total marrow irradiation (TMI).

TomoTherapy system showed a high stability of geometrical, mechanical and radiation parameters. The planning system allows to provide high quality therapeutic plans, which might be delivered with high accuracy due to the application of MVCT imaging. Only low grade acute toxicities were observed in patients who were treated in UCT during 2015-2016 years.

However, using TomoTherapy system raises some issues:

1. Long time beamlets calculating. The time of calculating may be reduced with using the VoLO system, which comprises a computer node with highperformance Graphics Processing Units (GPUs), coupled with Accuray's Non-Voxel Broad Beam (NVBB) algorithm.

2. TomoTherapy Contouring system has poor functionality and it is not adapted for automatic creation of any additional logical structures. Contouring system of other manufacturers is needed to create additional contours. At UCT MIM Contouring system is used. It allows to create outlines automatically, according to presets for different locations under local protocols. Similarly, the creation of presets for TPS accelerates the preparation before starting of plan optimization.

\section{REFERENCES}

1. T.Piotrowski, M. Skórska, A. Jodda, R. Ryczkowski, "Tomotherapy - a different way of dose delivery in radiotherapy," Contemp Oncol (Pozn), vol. 16, no. 1, pp. 16 - 25, Jan. 2012.

DOI: $10.5114 /$ wo.2012.27332

PMid: 23788850

PMCid: PMC 3687380

2. T. R. Mackie, T. W. Holmes, S. Swerdloff, P. Reckwerdt, J. O. Deasy, et al., "Tomotherapy: a new concept in the delivery of dynamic conformal radiotherapy," Med. Phys., vol. 20, no. 6, pp. 1709-1719, Nov-Dec. 1993. DOI: $10.1118 / 1.596958$

PMid: 8309444

3. T. R. Mackie et al., "Image guidance for precise conformal radiotherapy," Int. J. Radiat. Oncol. Biol. Phys., vol. 56, no. 1, pp. 89-105, May 2003. DOI: 10.1016/So360-3016(03)00090-7 PMid: 12694827

4. L. B. Marks, E. D. Yorke et al., "Use of normal tissue complication probability models in the clinic," Int. $J$. Radiat. Oncol. Biol. Phys., vol. 76, no. 3, pp. 10 - 19, Mar. 2010.

DOI: 10.1016/j.ijrobp.2009.07.1754

PMid: 20171502 PMCid: PMC4041542

5. Langen et al., "QA for helical tomotherapy: Report of the AAPM Task Group 148," Med. Phys., vol. 37, no. 9, pp. $4817-4853$, Sep. 2010

DOI: $10.1118 / 1.3462971$ PMid: 20964201

6. "Response assessment in solid tumours (RECIST): Version 1.1 and Supporting Papers," European Journal of Cancer, vol. 45, no. 2, pp. 225 - 310, Jan. 2009. Retrieved from: http://www.sciencedirect.com/science/journal/095980 49/45/2?sdc $=1$

Retrieved on: Jan. 24, 2017 